Conclusions: Setting up and effective utilization of any new investigation modalities has its own challenges. Our experience shows that it takes multiple iteration of the shifting process, along with initial training session and mock drill, proper education of neurosurgical technicians and nursing staff, and meticulous data collection and auditing to analyze and smoothen the workflow.

\section{A0038 A Rare Case of Cephalic Tetanus: Diagnostic Dilemma}

Rahul Ghiya, ${ }^{1}$ Bhibukalyani Das, ${ }^{1}$ Indranil Ghosh ${ }^{1}$

${ }^{1}$ Department of Neuro-Critical Care, Institute of Neurosciences, Kolkata, West Bengal, India

Background: Tetanus is an acute toxemic illness caused by soluble exotoxin of Clostridium tetani. Localized tetanus, especially the cephalic component, is extremely rare.

Case Description: The case of a 64-year-old woman with cephalic tetanus is described who went through a stormy hospital stay but ultimately went home in a stable condition. She was admitted with deviation of mouth to the left and slurring of speech without any loss of consciousness or muscle weakness. Next day she was complaining of dysphagia and choking. She was started on methylprednisolone and immunoglobulin suspecting a diagnosis of acute infective demyelinating polyneuropathy with bulbar involvement. At this time, all investigations including CSF analysis and MRI were normal. Over the next day, her symptoms increased and she developed trismus with sudden laryngospasm and respiratory arrest. She was intubated after administration of succinylcholine and ventilated under sedation. Over the next 2 days, she continued to develop autonomic dysfunction and was administered anti-tetanus immunoglobulin 500 IU intramuscularly. She underwent tracheostomy after which she again received 2,000 IU of anti-tetanus immunoglobulin. After this she received magnesium infusion to target a serum magnesium of 3 to $4 \mathrm{mg} / \mathrm{dL}$. She continued to receive magnesium till it was more than $4 \mathrm{mg} / \mathrm{dL}$. As trismus and autonomic dysfunction continued, she was given diazepam $5 \mathrm{mg}$ twice daily orally and dexmedetomidine infusion. Gradually, she was weaned off, and dose of diazepam was increased to $10 \mathrm{mg}$ thrice daily. As a last resort, she was administered intrathecal anti-tetanus immunoglobulin $250 \mathrm{IU}$. After this she showed gradual improvement in her symptoms and was decannulated. Thus, after almost 4 weeks, she was fit for discharge.

Conclusions: Cephalic tetanus is characterized by frequent laryngeal spasms with danger of death from asphyxia. It was a difficult case with lots of diagnostic dilemma, successfully managed at our institute.

\author{
A0039 Effect of Perioperative Blood Pressure on \\ Neurological Outcome in Patients Undergoing Clipping \\ following Aneurysmal Subarachnoid Hemorrhage \\ Shailesh Gupta, ${ }^{1}$ Prachi Agarwal, ${ }^{1}$ Nidhi Panda, \\ Kiran Jangra, ${ }^{1}$ Sivashanmugam S. Dhandapani, ${ }^{2}$ \\ Hemant Bhagat ${ }^{1}$ \\ ${ }^{1}$ Department of Anaesthesia and Intensive Care, Postgraduate \\ Institute of Medical Education and Research, Chandigarh, India \\ ${ }^{2}$ Department of Neurosurgery, Postgraduate Institute of Medical \\ Education and Research, Chandigarh, India
}

Background: It is postulated that elevated blood pressure (BP) is a homeostatic response to elevated intracranial pressure serving to maintain cerebral blood flow. Low $\mathrm{BP}$ results in cerebral hypoperfusion, which may aggravate ischemic injury. Studies to define the optimum BP associated with good neurological outcome are lacking. Therefore, we sought to observe the effect of perioperative blood pressure on long-term neurological outcome of patients with aneurysmal SAH.

Materials and Methods: After Institute Ethics Committee approval and written informed consent from the patients or their nearest kin, 338 patients with SAH of all grades and age more than 18 years scheduled to undergo surgery were included in the study. The systolic, diastolic and mean blood pressures were recorded at admission, preoperatively and intraoperatively. Postoperative blood pressures were recorded till ICU stay of the patient.

Results: Higher values of SBP, DBP, and mean arterial pressure (MAP) at the time of admission and in the preoperative period were associated with favorable neurological outcome at 3 months. There was no effect of intraoperative BP on the postoperative long-term neurological outcome. In the early postoperative period, patients with higher SBP, DBP, and MAP were associated with unfavorable neurological outcome. However, multiple logistic regression analysis did not demonstrate the effect of perioperative BP as an independent risk factor for long-term neurological outcome in patients with aneurysmal SAH.

Conclusions: The perioperative blood pressure is not an independent predictor of long-term neurological outcome in patients undergoing aneurysmal neck clipping following $\mathrm{SAH}$. Since admission, preoperative and early postoperative BPs have the potential to affect the outcome, BP should be meticulously observed and maintained within the normal physiological limits.

\section{A0040 Evaluation of Cerebral Perfusion Pressure and Cerebral Blood Flow Velocities in Different Head Positions Using Transcranial Doppler in Neurosurgical Patients Nisha Basker, ${ }^{1}$ Sethuraman Manikandan, ${ }^{1}$ Mathew Abraham² ${ }^{1}$ Department of Anaesthesia, Sree Chitra Tirunal Institute for Medical Sciences and Technology, Trivandrum, Kerala, India ${ }^{2}$ Division of Neuroanaesthesiology, Department of Neurosurgery, Sree Chitra Tirunal Institute for Medical Sciences and Technology, Trivandrum, Kerala, India}

Background: Different head positions in neurosurgical patients in the postoperative unit may affect cerebral perfusion pressure. The primary aim of this study was to investigate the effects of various head positions on the cerebral blood flow velocities by transcranial Doppler (TCD) in these patients.

Materials and Methods: This study with observational, prospective repeated measures was designed to measure bilateral MCA (middle cerebral artery) flow velocities, pulsatility index using TCD at different head positions in 20 patients who underwent cranial surgery admitted in the NSICU within 24 hours of surgery. The data collection 